TRANSACTIONS OF THE

AMERICAN MATHEMATICAL SOCIETY

Volume 353 , Number 6 , Pages $2427-2440$

S 0002-9947(01)02728-3

Article electronically published on January 16, 2001

\title{
EQUIVARIANT SURGERY \\ WITH MIDDLE DIMENSIONAL SINGULAR SETS. II: EQUIVARIANT FRAMED COBORDISM INVARIANCE
}

\author{
MASAHARU MORIMOTO
}

Dedicated to Professor Mamoru Mimura on his sixtieth birthday

\begin{abstract}
Let $G$ be a finite group and let $f: X \rightarrow Y$ be a degree 1, $G$-framed map such that $X$ and $Y$ are simply connected, closed, oriented, smooth manifolds of dimension $n=2 k \geqq 6$ and such that the dimension of the singular set of the $G$-space $X$ is at most $k$. In the previous article, assuming $f$ is $k$ connected, we defined the $G$-equivariant surgery obstruction $\sigma(f)$ in a certain abelian group. There it was shown that if $\sigma(f)=0$ then $f$ is $G$-framed cobordant to a homotopy equivalence $f^{\prime}: X^{\prime} \rightarrow Y$. In the present article, we prove that the obstruction $\sigma(f)$ is a $G$-framed cobordism invariant. Consequently, the $G$-surgery obstruction $\sigma(f)$ is uniquely associated to $f: X \rightarrow Y$ above even if it is not $k$-connected.
\end{abstract}

\section{INTRODUCTION}

This article is a continuation of 3 . Let $G$ be a finite group and $X$ a smooth $G$-manifold. The term $G$-equivariant surgery or simply $G$-surgery will refer to $G$ surgery on the free part of the $G$-space $X$, namely where every nontrivial element of $G$ acts without fixed points. Thus our $G$-surgery does not change the $G$-singular set

$$
\operatorname{Sing}(G, X)=\bigcup_{g \in G \backslash\{1\}} X^{g},
$$

where $X^{g}=\{x \in X \mid g x=x\}$.

Throughout the paper, $R$ denote the ring of integers $\mathbb{Z}$, its localization $\mathbb{Z}_{(p)}$ at a prime $p$, or the ring of rational numbers $\mathbb{Q}$. Associated to a degree $1, k$-connected $G$-framed map $f: X^{n} \rightarrow Y^{n}$ with $n=2 k$ such that the dimension of the $G$ singular set of $X$ is at most $k$, we [3] defined an element $\sigma(f)$ in an abelian group $W(G, Y ; R)$ such that if $\sigma(f)=0$ then $f$ can be converted to a $k$-connected, $R$ homology equivalence $f^{\prime}: X^{\prime} \rightarrow Y$ by $G$-surgery on the free part of $X$. The group $W(G, Y ; R)$ is a generalization of Wall's group $L_{n}^{h}(R[G])$, and ingredients for the generalization are quadratic and symmetric form parameters and positioning maps. Here $R[G]$ is the group ring of $G$ with coefficients in $R$. The purpose of this paper

Received by the editors October 12, 1999.

2000 Mathematics Subject Classification. Primary 57R67, 57R91, 19 G24.

Key words and phrases. Equivariant surgery, surgery obstruction, cobordism invariant, quadratic module.

Research partially supported by Max-Plank-Institut für Mathematik in Bonn and also by Grant-in-Aid for Scientific Research. 
is to prove that the obstruction $\sigma(f)$ is a $G$-framed cobordism invariant, namely Theorem 3.2. Moreover, the theorem enables us to define a unique element $\sigma(f)$ in $W(G, Y ; R)$ for a degree $1, G$-framed map $f$ even if $f$ is not $k$-connected.

A word about motivation and applications of this study is in order. So as to construct interesting smooth $G$-actions on manifolds, especially disks and spheres, we are first required to construct $G$-normal maps. As in the ordinary surgery theory, we can construct $G$-normal maps using the $G$-transverse regularity argument in 9. A $G$-normal map $f: X \rightarrow Y$ determines a stepwise surgery obstruction $\sigma\left(\operatorname{res}_{K}^{G} f^{P}\right)$ in $W\left(K, \operatorname{res}_{K}^{G} Y^{P} ; \mathbb{Z}_{(p)}\right)$ for a prime $p$, a $p$-subgroup $P \leqq G$ and $K=$ $N_{G}(P)$. Here if $P=\{1\}$ then we regard $\mathbb{Z}_{(p)}$ as $\mathbb{Z}$. So, second, we are required to modify or originally construct $G$-normal maps $f: X \rightarrow Y$ so that all surgery obstructions $\sigma\left(\operatorname{res}_{K}^{G} f^{P}\right)$ are trivial. A $G$-framed cobordism invariance theorem of surgery obstructions is useful to obtain the triviality of surgery obstructions as follows. For simplicity, we assume that all surgery obstructions $\sigma\left(\operatorname{res}_{K}^{G} f^{P}\right)$ are trivial if $P \neq\{1\}$. Then our eyes are focused on the surgery obstruction $\sigma(f)$ in $W(G, Y ; \mathbb{Z})$. In various cases, one can show that the canonical homomorphism

$$
\text { res }: W(G, Y ; \mathbb{Z}) \rightarrow \bigoplus_{H} W\left(H, \operatorname{res}_{H}^{G} Y ; \mathbb{Z}\right)
$$

where $H$ runs over an appropriate set of subgroups — such as a set containing all hyperelementary subgroups and all dihedral subgroups — of $G$, is injective (cf. [2] and [7]). Using the construction of [9], for most subgroups $H$, there often exist $H$-normal cobordisms between $\operatorname{res}_{H}^{G} f$ and $\operatorname{res}_{H}^{G} i d_{Y}$. In this case, Theorem 3.2 guarantees $\sigma\left(\operatorname{res}_{H}^{G} f\right)=\sigma\left(\operatorname{res}_{H}^{G} i d_{Y}\right)=0$. Along this strategy, we can construct various smooth actions on disks and spheres for nontrivial perfect groups and nilpotent Oliver groups. In fact, we can prove that there exist smooth one fixed point $A_{5}$-actions on $S^{n}$ if and only if $n \geqq 6([2])$, which provides the smooth version of the Buchdahl-Kwasik-Schultz' theorem [4, Theorem II.5]. This fact shows the fundamental significance of Theorem 3.2, although the outlook of the theorem is complicated.

Theorem 1.1. Let $G$ be a finite group and $Y$ a closed, 1-connected, oriented, smooth $G$-manifold of even dimension $n=2 k \geqq 6$. Suppose that (1.1.1)-(1.1.3) below hold.

(1.1.1) $\operatorname{dim} Y^{g} \leqq k$ for all $g \in G \backslash\{1\}$.

(1.1.2) If $\operatorname{dim} Y^{H}=k$ for some subgroup $H \leqq G$, then $|H|=2$ and $Y^{H}$ is connected and oriented so that each $g: Y^{H} \rightarrow Y^{g H g^{-1}}(g \in G)$ is orientation preserving.

(1.1.3) $\operatorname{dim}\left(Y^{H} \cap Y^{K}\right) \leqq k-2$ whenever $\operatorname{dim} Y^{H}=k$ and $\operatorname{dim} Y^{K}=k-1$ (H, $K \leqq G)$.

Let $X$ be a closed, connected, oriented, smooth $G$-manifold satisfying (1.1.1)-(1.1.3) above, $\xi$ and $\eta$ real $G$-vector bundles over $Y$ and $f=\left(f: X \rightarrow Y, b: T(X) \oplus f^{*} \eta \rightarrow\right.$ $\left.f^{*}(\xi \oplus \eta)\right)$ a degree 1, G-framed map. Suppose that

(1.1.4) if $H \leqq G$ has order 2 and if $\operatorname{dim} X^{H} \geqq k-1$ or $\operatorname{dim} Y^{H} \geqq k-1$, then $\operatorname{dim} X^{H}=\operatorname{dim} \bar{Y}^{H}$

(1.1.5) if $R=\mathbb{Z}$ then $f^{H}: X^{H} \rightarrow Y^{H}$ is a $\mathbb{Z}$-homology equivalence for any hyplerelementary subgroup $H \neq\{1\}$ of $G$;

(1.1.6) if $R=\mathbb{Z}_{(p)}$ (p a prime) then $f^{P}: X^{P} \rightarrow Y^{P}$ is a $\mathbb{Z}_{(p) \text {-homology }}$ equivalence for any $p$-subgroup $P \neq\{1\}$ of $G$; and 
(1.1.7) if $R=\mathbb{Q}$ then $\chi\left(X^{g}\right)=\chi\left(Y^{g}\right)$ for any $g \in G \backslash\{1\}$, where $\chi(-)$ denotes the Euler characteristic.

Then one can associate to $\boldsymbol{f}$ an element $\sigma(\boldsymbol{f})$ in the abelian group $W(G, Y ; R)$ of [3, Theorem 1.1] such that $\sigma(\boldsymbol{f})=0$ if and only if $\boldsymbol{f}$ is $G$-framed cobordant rel. $\bigcup X^{g}$ to a degree $1, k$-connected, G-framed map $f^{\prime}=\left(f^{\prime}: X^{\prime} \rightarrow Y, b^{\prime}\right.$ : $g \in G \backslash\{1\}$

$\left.T\left(X^{\prime}\right) \oplus{f^{\prime *}}^{*} \rightarrow{f^{\prime *}}^{*}(\xi \oplus \eta)\right)$ such that $f^{\prime}: X^{\prime} \rightarrow Y$ is an $R$-homology equivalence.

Theorem 1.1 will be deduced from a slight generalization Theorem 3.3 of it.

Remark 1.2. (1) If $\sigma(\boldsymbol{f})=0$ then a $G$-framed map $\boldsymbol{f}^{\prime}$ satisfying the properties in Theorem 1.1 and a $G$-framed cobordism between $f$ and $\boldsymbol{f}^{\prime}$ can be obtained by $G$-surgery of dimension $\leqq k$ on the free part of $X$.

(2) If $f: X \rightarrow Y$ is $k$-connected then the $\sigma(f)$ in the theorem is same as in [3] Theorem 1.1].

The reader can refer to [8] for the $G$-framed cobordism invariance of $G$-surgery obstructions under the hypothesis that $\operatorname{dim} \operatorname{Sing}(G, X) \leqq k-1$. In the case where $G=\mathbb{Z}_{2}$, the cyclic group of order 2, Dovermann [5] and Dovermann-Schultz [6] described $\mathbb{Z}_{2}$-surgery obstructions in different ways and proved the equivariant normal cobordism invariance of the obstructions. Since $\widetilde{K}_{0}\left(\mathbb{Z}\left[\mathbb{Z}_{2}\right]\right)=0$, Theorem 3.3 gives results corresponding to $\left[\underline{6}\right.$, Theorems $\mathrm{A}_{0}$ and $\left.\mathrm{B}\right]$. It is remarkable that Dovermann-Schultz [6] allowed the case where the $\mathbb{Z}_{2}$-fixed point set of the middle dimension is not orientable. Readers would wonder if the results in the present paper could be generalized to those allowing nonorientable middle dimensional fixed point sets, and moreover allowing nonconnected middle dimensional fixed point sets. We ([1]) know that such generalization is possible, but it needs a lot of formalities to describe. It would also be interesting to formalize $G$-surgery theory for simple $G$-homotopy equivalences on compact $G$-manifolds with middle dimensional fixed point sets. Readers familiar with the ordinary surgery theory [10] could develop such $G$-surgery theory without difficulty using the arguments in [3] and this paper, although they have to modify the $G$-surgery obstruction groups. Since we have written the present paper for specific applications, we postpone describing them until future papers.

The remainder of the paper is organized as follows. Section 2 is devoted to preparation for Sections 3 and 4. The $G$-framed cobordism invariance of the $G$ surgery obstruction is presented as Theorem 3.2. The main body of its proof is stated as Lemma 3.1. The generalization, Theorem 3.3, mentioned above is obtained as a corollary to Theorem 3.2. Section 3 consists of these three results. We prove Lemma 3.1 in Section 4, and a key method of the proof is equivariant handle subtraction.

Acknowledgements. Most of this paper was written during author's stay at the Max-Planck-Institut für Mathematik in Bonn. He would like to express his gratitude to the institute for its warm hospitality.

\section{Preliminary}

In the current article, $n=2 k$ is an even integer $\geqq 6$, and a manifold (resp. an action on a manifold) means a smooth manifold (resp. a smooth action) unless otherwise specified. Let $((\operatorname{proj}))$ (resp. $(($ s-free $)),(($ free $)))$ denote the category of 
all finitely generated projective (resp. stably free, free) $R[G]$-modules. Let $\mathcal{C}$ denote one of these three categories.

Let $X$ be a $G$-CW complex (e.g. $G$-manifold, $G$-simplicial complex). For a point $x \in X$ and a subgroup $H$ of $G$, let $H_{x}$ denote the isotropy subgroup at $x$ in the $H$-space $\operatorname{res}_{H}^{G} X$. Set

$$
\begin{aligned}
& X^{H}=\operatorname{Fix}(H, X)=\left\{x \in X \mid G_{x} \supseteqq H\right\}, \\
& X^{>H}=\operatorname{Fix}_{G}(>H, X)=\left\{x \in X \mid G_{x} \supsetneqq H\right\}, \\
& X^{=H}=\operatorname{Fix}_{G}(=H, X)=\left\{x \in X \mid G_{x}=H\right\}, \\
& \operatorname{Free}(H, X)=\left\{x \in X \mid H_{x}=1\right\}, \\
& \operatorname{Sing}(H, X)=\left\{x \in X \mid H_{x} \neq\{1\}\right\} .
\end{aligned}
$$

If $g \in G$ then set $X^{g}=X^{\langle g\rangle}$. For a subspace $Z \subseteq X$, we define the subgroup

$$
\rho_{X}^{G}(Z):=\bigcap_{x \in Z} G_{x} .
$$

Let $\pi_{0}\left(X^{H}\right)$ denote the set of all connected components $\gamma$ of $X^{H}$. The underlying space of $\gamma$ is denoted by $X_{\gamma}$. If we need to emphasize the group $H$ then we use $X_{\gamma}^{H}$ instead of $X_{\gamma}$. For a nonnegative integer $\ell$, let $\pi_{0}\left(X^{H}, \ell\right)$ denote the subset of $\pi_{0}\left(X^{H}\right)$ consisting of all $\gamma$ with $\operatorname{dim} X_{\gamma}=\ell$. We set

$$
\begin{aligned}
& \Pi(G, X)=\coprod_{H \leqq G} \pi_{0}\left(X^{H}\right), \\
& \Pi(G, X, \ell)=\coprod_{H \leqq G} \pi_{0}\left(X^{H}, \ell\right) .
\end{aligned}
$$

For $\gamma \in \Pi=\Pi(G, X)$ such that $\gamma \in \pi_{0}\left(X^{H}\right)$, define $\rho(\gamma)=\rho_{\Pi}(\gamma):=H$. Clearly, $\rho_{\Pi}(\gamma) \leqq \rho_{X}^{G}\left(X_{\gamma}\right)$. The set $\Pi$ has the canonical $G$-action such that $X_{g \gamma}=g X_{\gamma}$ and $\rho(g \gamma)=g \rho(\gamma) g^{-1}$, and $\Pi(G, X, \ell)$ is a $G$-invariant subset of $\Pi$. Let $\Phi: \Pi \rightarrow \Pi$ denote the map $\gamma \mapsto \beta$ such that $\rho(\beta)=\rho_{X}^{G}\left(X_{\gamma}\right)$ and $X_{\beta}=X_{\gamma}$. Define

$$
\begin{aligned}
& \Theta(G, X):=\operatorname{Im}(\Phi), \\
& \Theta(G, X, \ell):=\Theta(G, X) \cap \Pi(G, X, \ell) .
\end{aligned}
$$

Let $\boldsymbol{M} \boldsymbol{n} \boldsymbol{f}_{\mathrm{cp}}^{n}(G)_{\ell}\left(\right.$ resp. $\left.\boldsymbol{M} \boldsymbol{n} \boldsymbol{f}_{\mathrm{cl}}^{n}(G)_{\ell}\right)$ denotes the family of all compact (resp. closed), $\ell$-connected, oriented $G$-manifolds of dimension $n$. Each $G$-manifold $X \in$ $\boldsymbol{M n} \boldsymbol{f}_{\mathrm{cp}}^{n}(G)_{0}$ has orientation homomorphism $w_{X}: G \rightarrow\{ \pm 1\}$ such that $w_{X}(g)$ $=1$ (resp. -1$)$ for orientation preserving (resp. reversing) $g \in G$.

Let $G(2)$ denote the set of all elements of order 2 in $G$. (The identity element does not belong to $G(2)$.) In the case $n=2 k$, let $\boldsymbol{M} \boldsymbol{n} \boldsymbol{f}_{\mathrm{cp}, \mathrm{sg}}^{n}(G)_{\ell}\left(\operatorname{resp} . \boldsymbol{M} \boldsymbol{n} \boldsymbol{f}_{\mathrm{cl}, \mathrm{sg}}^{n}(G)_{\ell}\right)$ denote the family of all $X \in \boldsymbol{M} \boldsymbol{n} \boldsymbol{f}_{\mathrm{cp}}^{n}(G)_{\ell}$ (resp. $\left.\boldsymbol{M} \boldsymbol{n} \boldsymbol{f}_{\mathrm{cl}}^{n}(G)_{\ell}\right)$ satisfying the following properties:

(2.1.1) $\operatorname{dim} \operatorname{Sing}(G, X) \leqq k$.

(2.1.2) $\left|\pi_{0}\left(X^{H}, k\right)\right| \leqq 1$ for any $H \leqq G$.

(2.1.3) If $\gamma \in \Theta(G, X, k)$ then $|\rho(\gamma) \cap G(2)|=1$.

(2.1.4) If $\gamma \in \Theta(G, X, k)$ and $\delta \in \Theta(G, X, k-1)$ then $\operatorname{dim}\left(X_{\gamma} \cap X_{\delta}\right) \leqq k-2$.

(2.1.5) All submanifolds $X_{\gamma}^{H}(\gamma \in \Theta(G, X, k)$ and $H=\rho(\gamma))$ are oriented so that each $g \in G$ acts as an orientation preserving diffeomorphism $X_{\gamma}^{H} \rightarrow X_{g \gamma}^{g H g^{-1}}$. 
If $n$ is an even integer $\geqq 6$, say $n=2 k$, and $X$ belongs to $\boldsymbol{M} \boldsymbol{n} \boldsymbol{f}_{c p, s g}^{n}(G)_{0}$, then set

$$
\begin{aligned}
& \lambda=\lambda_{X}:=(-1)^{k}, \\
& Q=Q(G, X):=\left\{g \in G(2) \mid \operatorname{dim} X^{g}=k-1\right\}, \\
& S=S(G, X):=\left\{g \in G(2) \mid \operatorname{dim} X^{g}=k\right\} .
\end{aligned}
$$

The set $S$ is identified with $\Theta(G, X, k)$ via the map

$$
\Theta(G, X, k) \rightarrow S(G, X) ; \gamma \longmapsto g \text { such that } g \in \rho(\gamma)
$$

Depending on $X$ and the choice of $R=\mathbb{Z}, \mathbb{Z}_{(p)}$ ( $p$ a prime) or $\mathbb{Q}$, let $\boldsymbol{A}$ denote the tuple

$$
\left(R, G, Q, S, \lambda, w_{X}\right)
$$

The group ring $R[G]$ is equipped with the anti-involution ${ }^{-}$such that

$$
\overline{\sum_{g \in G} c_{g} g}=\sum_{g \in G} w_{X}(g) c_{g} g^{-1} \quad\left(c_{g} \in R\right) .
$$

If $a \in G$ then let $\varepsilon_{a}$ denote the map $R[G] \rightarrow R$ or $R / 2 R[G] \rightarrow R / 2 R$ defined by

$$
\varepsilon_{a}\left(\sum_{g \in G} c_{g} g\right)=c_{a} \quad\left(c_{g} \in R \text { or } R / 2 R\right) .
$$

The choice of $R$ or $R / 2 R$ will be clear from the context. For a subset $T$ of $G, R[T]$ denotes the $R$-submodule of $R[G]$ which is generated by all elements in $T$. Let $\Lambda=\Lambda(G, X ; R)$ denote the quadratic form parameter defined by

$$
\Lambda=\min ^{\lambda}(R[G])+R[Q], \text { where } \min ^{\lambda}(R[G])=\langle x-\lambda \bar{x} \mid x \in R[G]\rangle_{R} .
$$

A special quadratic $(\boldsymbol{A}, \Theta(G, X, k))$-module $\boldsymbol{M}$ is a tuple $(M, B, q, \alpha)$ of

$M$ a finitely generated, projective $R[G]$-module,

$B: M \times M \rightarrow R[G]$ a nonsingular $\lambda$-Hermitian form,

$q: M \rightarrow R[G \backslash S] / \Lambda$ an $\boldsymbol{A}$-quadratic form and

$\alpha: \Theta(G, X, k) \rightarrow M \quad$ a $G$-map

satisfying the conditions:

(2.2.1) $B$ is biadditive,

(2.2.2) $B(a x, b y)=b B(x, y) \bar{a}$,

(2.2.3) $B(x, y)=\lambda \overline{B(y, x)}$

(2.2.4) $q(g x)=g q(x) \bar{g}$ and $q(r x)=r^{2} q(x)$ in $R[G \backslash S] / \Lambda=R[G] /(\Lambda+R[S])$,

(2.2.5) $q(x+y)-q(x)-q(y)=B(x, y)$ in $R[G \backslash S] / \Lambda=R[G] /(\Lambda+R[S])$,

(2.2.6) $\widetilde{q(x)}+\lambda \widetilde{q(x)}=B(x, x)$ in $R[G \backslash S]=R[G] / R[S]$ and

(2.2.7) $\left[\varepsilon_{1}(B(\alpha(s)-x, s x))\right]=0$ in $R / 2 R$,

for all $x, y \in M, a, b \in R[G], g \in G, r \in R$ and $s \in S$ (see [3. pp.278-279]), where $\widetilde{q(x)}$ is a lifting of $q(x)$.

If $\boldsymbol{M}=(M, B, q, \alpha)$ is a special quadratic $(\boldsymbol{A}, \Theta(G, X, k))$-module then let $-\boldsymbol{M}$ stand for the module $(M,-B,-q,-\alpha)$. Let $\boldsymbol{S} \boldsymbol{Q}(\boldsymbol{A}, \Theta(G, X, k))_{\mathcal{C}}$ denote the category of all special quadratic $(\boldsymbol{A}, \Theta(G, X, k))$-modules whose underlying modules are in $\mathcal{C}$.

For a $G$-map $f: X \rightarrow Y$, we set

$$
K_{\ell}(X ; R)=K_{\ell}(f ; R):=\operatorname{Ker}\left[f_{*}: H_{\ell}(X ; R) \rightarrow H_{\ell}(Y ; R)\right] .
$$


It is quite important to see whether the $R[G]$-module $K_{\ell}(X ; R)$ is stably free or projective. To this end, let us define the following conditions on a $G$-map $f: X \rightarrow$ $Y$.

$\mathcal{F}_{1}$-Condition: $\quad \chi\left(X^{g}\right)=\chi\left(Y^{g}\right)$ for any $G \backslash\{1\}$. (Here $\chi(-)$ denotes the Euler characteristic.)

$\mathcal{F}_{p^{-} \text {Condition: }} \quad f^{P}: X^{P} \rightarrow Y^{P}$ is a $\mathbb{Z}_{(p)}$-homology equivalence for any $p$ subgroup $P \neq\{1\}$ of $G$.

$\mathcal{P}_{0}$-Condition: $f^{P}: X^{P} \rightarrow Y^{P}$ is a $\mathbb{Z}_{(p)}$-homology equivalence for any prime $p$ dividing $|G|$ and any $p$-subgroup $P \neq\{1\}$ of $G$.

$\mathcal{F}_{0}$-Condition: $f^{H}: X^{H} \rightarrow Y^{H}$ is a $\mathbb{Z}$-homology equivalence for any hyperelementary subgroup $H \neq\{1\}$ of $G$.

Remark 2.3. Let $R=\mathbb{Z}_{(m)}$ for $m=0,1$ or a prime, where $\mathbb{Z}_{(0)}=\mathbb{Z}$ and $\mathbb{Z}_{(1)}=\mathbb{Q}$. Let $X$ and $Y$ be finite $G$-CW complexes (e.g. compact $G$-manifolds), let $\ell$ be a positive integer, and let $f: X \rightarrow Y$ be an $\ell$-connected $G$-map such that $f_{*}$ : $H_{s}(X ; R) \rightarrow H_{s}(Y ; R)$ is an isomorphism for every $s \neq \ell$ and $K_{\ell}(X ; R)$ is $\mathbb{Z}$-torsion free. Then the following hold.

(2.3.1) If $f$ satisfies the $\mathcal{F}_{m}$-Condition, then $K_{\ell}(X ; R)$ is stably free over $R[G]$.

(2.3.2) If $R=\mathbb{Z}$ and if $f$ satisfies the $\mathcal{P}_{0}$-Condition, then $K_{\ell}(X ; \mathbb{Z})$ is projective over $\mathbb{Z}[G]$. If furthermore $\widetilde{K}_{0}(\mathbb{Z}[G])=0$, then $K_{\ell}(X ; \mathbb{Z})$ is stably free over $\mathbb{Z}[G]$.

Let $X, X^{\prime} \in \boldsymbol{M} \boldsymbol{n} \boldsymbol{f}_{\mathrm{cl}, \mathrm{sg}}^{n}(G)_{0}$ and $Y \in \boldsymbol{M} \boldsymbol{n} \boldsymbol{f}_{\mathrm{cl}}^{n}(G)_{1}$. Let $\boldsymbol{f}=(f: X \rightarrow Y$, $\left.b: T(X) \oplus f^{*} \eta \rightarrow f^{*}(\xi \oplus \eta)\right)$ and $f^{\prime}=\left(f^{\prime}: X^{\prime} \rightarrow Y, b^{\prime}: T\left(X^{\prime}\right) \oplus f^{\prime *} \eta \rightarrow\right.$ $\left.f^{\prime *}(\xi \oplus \eta)\right)$ be degree 1, $G$-framed maps for $G$-vector bundles $\xi$ and $\eta$ over $Y$ such that $\eta \supseteqq \varepsilon_{Y}\left(\mathbb{R}^{n+1}\right)$ (cf. [3, p.286]). Let $I$ denote the closed unit interval $[0,1]$ and $p_{Y}: I \times Y \rightarrow Y$ the canonical projection. Let $\boldsymbol{F}=(F:(W, \partial W) \rightarrow$ $\left.(I \times Y, \partial I \times Y), B: T(W) \oplus\left(p_{Y} \circ F\right)^{*} \eta \rightarrow\left(p_{Y} \circ F\right)^{*}\left(\varepsilon_{Y}(\mathbb{R}) \oplus \xi \oplus \eta\right)\right)$ be a $G$-framed cobordism between $f$ and $f^{\prime}$. In particular, $W$ is an oriented cobordism between $X$ and $X^{\prime}$, i.e. $\partial W=(-X) \amalg X^{\prime}$.

Definition 2.4. A $G$-framed cobordism $\boldsymbol{F}$ between $\boldsymbol{f}$ and $\boldsymbol{f}^{\prime}$ is said to be admissible if the following conditions (2.4.1)-(2.4.3) are fulfilled.

(2.4.1) $\operatorname{dim} \operatorname{Sing}(G, W) \leqq k+1$.

(2.4.2) The inclusion $j: X \rightarrow W$ (resp. $j^{\prime}: X^{\prime} \rightarrow W$ ) induces bijections $\Pi(G, X, k) \rightarrow \Pi(G, W, k+1)$ and $\Pi(G, X, k-1) \rightarrow \Pi(G, W, k)\left(\right.$ resp. $\Pi\left(G, X^{\prime}, k\right) \rightarrow$ $\Pi(G, W, k+1)$ and $\left.\Pi\left(G, X^{\prime}, k-1\right) \rightarrow \Pi(G, W, k)\right)$.

We use $W_{\gamma}$ for $W_{j_{\#} \gamma}$ (resp. also for $W_{j_{\#}^{\prime} \gamma}$ ) if $\ell=k-1$ or $k$ and $\gamma \in \Pi(G, X, \ell)$ (resp. $\Pi\left(G, X^{\prime}, \ell\right)$ ). Moreover we usually identify $\Theta\left(G, X^{\prime}, \ell\right)$ with $\Theta(G, X, \ell)$ via the induced maps when $\ell=k-1$ or $k$.

(2.4.3) For each $\gamma \in \Theta(G, X, k)=\Theta\left(G, X^{\prime}, k\right), W_{\gamma}$ is an oriented cobordism between $X_{\gamma}$ and $X_{\gamma}^{\prime}$.

Let $\boldsymbol{C o b}\left(G ; \boldsymbol{f}, \boldsymbol{f}^{\prime}\right)$ denote the family of all admissible, $G$-framed cobordisms between $\boldsymbol{f}$ and $\boldsymbol{f}^{\prime}$.

Lemma 2.5. Let $\boldsymbol{f}, \boldsymbol{f}^{\prime}$ and $\boldsymbol{F} \in \boldsymbol{C o b}\left(G ; \boldsymbol{f}, \boldsymbol{f}^{\prime}\right)$ be as above. Then $\boldsymbol{F}$ is $G$-framed cobordant rel. $\partial W \cup \operatorname{Sing}(G, W)$ (by $G$-surgery of dimension $\leqq k-1$ on $\operatorname{Free}(G, W) \backslash$ $\partial W)$ to a k-connected cobordism $\boldsymbol{F}^{\prime} \in \boldsymbol{C o b}\left(G ; \boldsymbol{f}, \boldsymbol{f}^{\prime}\right)$.

Proof. This is clear from the fact that $\operatorname{dim} \operatorname{Sing}(G, W)+(k-1)<\operatorname{dim} W$. 
Suppose that $\boldsymbol{f}, \boldsymbol{f}^{\prime}$ and $\boldsymbol{F}$ are $k$-connected. Then the next sequence for the coefficient ring $\mathbb{Z}$ is exact:

$$
0 \rightarrow K_{k+1}(W) \rightarrow K_{k+1}(W, \partial W) \stackrel{\partial}{\rightarrow} K_{k}(\partial W) \rightarrow K_{k}(W) \rightarrow K_{k}(W, \partial W) \rightarrow 0 .
$$

By the universal coefficient theorem, the sequence for the coefficient ring $R$ is also exact. Furthermore suppose $K_{k}(W, \partial W ; R)=0=K_{k+1}(W ; R)$ and regard $K_{k+1}(W, \partial W ; R)$ as a submodule of $K_{k}(\partial W ; R)$ via the connecting homomorphism $\partial$. The restriction $\partial \boldsymbol{F}$ of $\boldsymbol{F}$ to the boundary determines a special quadratic $(\boldsymbol{A}, \Theta(G, X, k))$-module

$$
\overline{\boldsymbol{M}}_{\partial \boldsymbol{F}}=\left(K_{k}(\partial W ; R), B_{\partial F}, q_{\partial \boldsymbol{F}}, \bar{\alpha}_{\partial F}\right)
$$

as follows. The map

$$
B_{\partial F}: K_{k}(\partial W ; R) \times K_{k}(\partial W ; R) \rightarrow R[G]
$$

is the equivariant intersection form and the map

$$
q_{\partial \boldsymbol{F}}: K_{k}(\partial W ; R) \rightarrow R[G \backslash S(G, X)]
$$

is the equivariant selfintersection form. Let

$$
\text { InterSec }_{\partial W}: K_{k}(\partial W ; R) \times K_{k}(\partial W ; R) \rightarrow R
$$

denote the ordinary intersection form. Then, by definition

$$
B_{\partial F}(x, y)=\sum_{g \in G} \operatorname{InterSec}_{\partial W}\left(x, g^{-1} y\right) g \quad\left(x, y \in K_{k}(\partial W ; R)\right) .
$$

We see immediately that $B_{\partial F}=\left(-B_{f}\right) \oplus B_{f^{\prime}}$. Similarly, $q_{\partial \boldsymbol{F}}=\left(-q_{\boldsymbol{f}}\right) \oplus q_{\boldsymbol{f}^{\prime}}$. The $G$-map $\bar{\alpha}_{\partial \boldsymbol{F}}: \Theta(G, X, k) \rightarrow K_{k}(\partial W ; R)$ is defined by

$$
\bar{\alpha}_{\partial F}(\gamma)=\pi_{\partial F}\left(j_{\partial W_{\gamma *}}\left(\partial\left[W_{\gamma}\right]\right)\right) \quad(\gamma \in \Theta(G, X, k)),
$$

where $\left[W_{\gamma}\right] \in H_{k+1}\left(W_{\gamma}, \partial W_{\gamma} ; R\right)$ is the orientation class, $j_{\partial W_{\gamma}}$ is the inclusion map $\partial W_{\gamma} \rightarrow \partial W$, and $\pi_{\partial F}: H_{k}(\partial W ; R) \rightarrow K_{k}(\partial W ; R)$ is the canonical projection. Thus we obtain the commutative diagram:

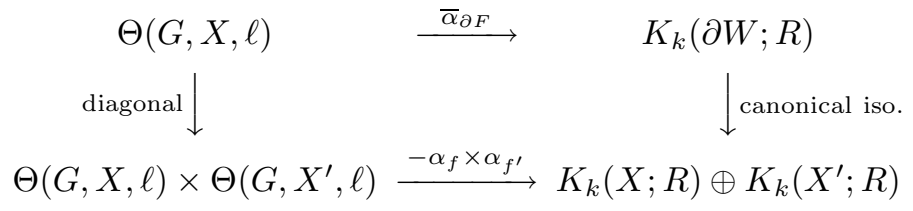

Remark 2.6. In [3, Lemma 7.1] we invoked $Y \in \boldsymbol{M} \boldsymbol{n} \boldsymbol{f}_{\mathrm{cl}, \mathrm{sg}}^{n}(G)_{1}$. But it is superfluous, and the condition $Y \in \boldsymbol{M} \boldsymbol{n} \boldsymbol{f}_{\mathrm{cl}}^{n}(G)_{1}$ suffices to obtain the conclusion in [3] Lemma 7.1], namely to guarantee $\boldsymbol{M}_{\boldsymbol{f}} \in \boldsymbol{S} \boldsymbol{Q}(\boldsymbol{A}, \Theta(G, X, k))_{\mathcal{C}}$ (for the definition of $\boldsymbol{M}_{\boldsymbol{f}}$, see [3, pp. 286-288]).

Lemma 2.7. Let $\boldsymbol{f}$ and $\boldsymbol{f}^{\prime}$ be as above. Suppose that $f: X \rightarrow Y$ and $f^{\prime}: X^{\prime} \rightarrow Y$ are $k$-connected. Let $\boldsymbol{F} \in \boldsymbol{C o b}\left(G ; \boldsymbol{f}, \boldsymbol{f}^{\prime}\right)$ be as above. Suppose that $F: W \rightarrow I \times Y$ is k-connected, and

$$
K_{k}(W, \partial W ; R)=0=K_{k+1}(W ; R) .
$$

Then the following hold.

(2.7.1) $\overline{\boldsymbol{M}}_{\partial \boldsymbol{F}}=-\boldsymbol{M}_{\boldsymbol{f}} \oplus \boldsymbol{M}_{\boldsymbol{f}^{\prime}}$.

(2.7.2) The submodule $K_{k+1}(W, \partial W ; R)$ of $K_{k}(\partial W ; R)$ includes $\operatorname{Im}\left(\bar{\alpha}_{\partial F}\right)$.

(2.7.3) The submodule $K_{k+1}(W, \partial W ; R)$ is totally isotropic in $\overline{\boldsymbol{M}}_{\partial \boldsymbol{F}}$; namely, $B_{\partial F}$ and $q_{\partial \boldsymbol{F}}$ are trivial on $K_{k+1}(W, \partial W ; R)$. 
Proof. (2.7.1): The conclusion immediately follows from $\partial W=(-X) \amalg X^{\prime}$ and the definition of $B_{\partial F}, q_{\partial \boldsymbol{F}}$ and $\bar{\alpha}_{\partial W}$.

(2.7.2): The conclusion is obtained from observation of the following commutative diagram:

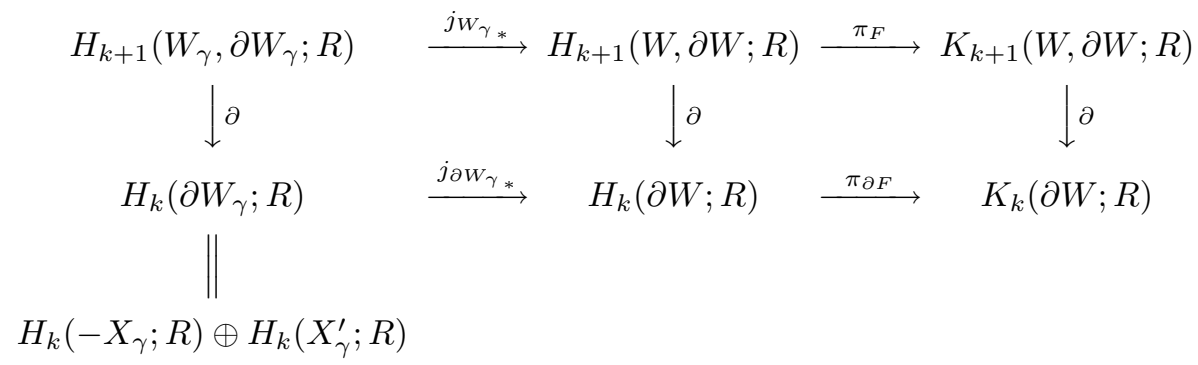

(2.7.3): Now we prove the last conclusion. Here the coefficient ring of homology groups is $R$. It is well known that the ordinary intersection form satisfies

$$
\operatorname{InterSec}_{\partial W}\left(K_{k+1}(W, \partial W), K_{k+1}(W, \partial W)\right)=0 .
$$

Since $K_{k+1}(W, \partial W)$ is $G$-invariant, it follows that

$$
B_{\partial F}\left(K_{k+1}(W, \partial W), K_{k+1}(W, \partial W)\right)=0 .
$$

Let us decompose $G$ to a disjoint union

$$
G=\{1\} \amalg G(2) \amalg G(\geqq 3)_{h} \amalg G(\geqq 3)_{h}{ }^{-1}
$$

such that $G(\geqq 3)_{h}$ is a subset of $G$ consisting of elements of order $\geqq 3$, and $G(\geqq 3)_{h}{ }^{-1}=\left\{g^{-1} \mid g \in G(\geqq 3)_{h}\right\}$. Let

$$
\begin{aligned}
& G^{\lambda}(2)=\left\{g \in G(2) \mid w_{X}(g)=-\lambda\right\}, \\
& G^{-\lambda}(2)=\left\{g \in G(2) \mid w_{X}(g)=\lambda\right\} .
\end{aligned}
$$

Then we can regard the map $q_{\partial \boldsymbol{F}}: K_{k}(\partial W) \rightarrow R[G \backslash S] / \Lambda$ as a map

$$
K_{k}(\partial W) \rightarrow R /(1-\lambda)[\{1\}] \oplus R / 2 R\left[G^{\lambda}(2) \backslash Q\right] \oplus R\left[G^{-\lambda}(2) \backslash S\right] \oplus R\left[G(\geqq 3)_{h}\right] .
$$

(cf. [3, p.274]). In the case where $g \in G(\geqq 3)_{h}$, we have $\varepsilon_{g}\left(q_{\partial W}(x)\right)=0$ for any $x \in K_{k+1}(W, \partial W)$, because

$$
\varepsilon_{g}\left(q_{\partial W}(x)\right)=\operatorname{InterSec}_{\partial W}\left(x, g^{-1} x\right) .
$$

Let $g \in\{1\} \cup\left(G^{\lambda}(2) \backslash Q\right) \cup\left(G^{-\lambda}(2) \backslash S\right)$. Then one has

$$
\varepsilon_{g}\left(q_{\partial \boldsymbol{F}}(x)\right)=\varepsilon_{g}\left(q_{\partial\left(\operatorname{res}_{\langle g\rangle}^{G} \boldsymbol{F}\right)}(x)\right)
$$

for any $x \in K_{k}(\partial W)=K_{k}\left(\operatorname{res}_{\langle g\rangle}^{G} \partial W\right)$. However, we have

$$
\begin{aligned}
\operatorname{dim}\left(\operatorname{Sing}_{\langle g\rangle}\left(\operatorname{res}_{\langle g\rangle}^{G} \partial W\right)\right)+(k+1) & \leqq\{(k-2)+1\}+(k+1) \\
& =2 k \\
& <\operatorname{dim}\left(\operatorname{res}_{\langle g\rangle}^{G} \partial W\right) .
\end{aligned}
$$

(In other words, $\operatorname{res}_{\langle g\rangle}^{G} \partial W$ satisfies the strong gap condition.) In this case, an argument similar to that of Wall [10, p.53, lines 7-11] proves that $q_{\operatorname{res}_{\langle g\rangle}^{G} \partial \boldsymbol{F}}(x)=0$ for all $x \in K_{k+1}\left(\operatorname{res}_{\langle g\rangle}^{G} W, \partial\left(\operatorname{res}_{\langle g\rangle}^{G} W\right)\right)$. Consequently we get $q_{\partial \boldsymbol{F}}(x)=0$ for all $x \in K_{k+1}(W, \partial W)$. 


\section{MAIN RESULTS}

Throughout this section let $\mathcal{C}$ denote the category ((proj)), ((s-free)) or $(($ free $))$. We begin the section with a lemma which is a key to Theorems 3.2 and 3.3.

Lemma 3.1. Let $X, X^{\prime} \in \boldsymbol{M} \boldsymbol{n} \boldsymbol{f}_{\mathrm{cl}, \mathrm{sg}}^{n}(G)_{0}$ and $Y \in \boldsymbol{M} \boldsymbol{n} \boldsymbol{f}_{\mathrm{cl}}^{n}(G)_{1}$ with $n=2 k \geqq 6$ even, and let $\boldsymbol{f}=\left(f: X \rightarrow Y, b: T(X) \oplus f^{*} \eta \rightarrow f^{*}(\xi \oplus \eta)\right)$ and $\boldsymbol{f}^{\prime}=\left(f^{\prime}: X^{\prime} \rightarrow\right.$ $\left.Y, b^{\prime}: T\left(X^{\prime}\right) \oplus{f^{\prime *}}^{*} \rightarrow f^{\prime *}(\xi \oplus \eta)\right)$ be degree $1, k$-connected, $G$-framed maps such that $K_{k}(X ; R)$ and $K_{k}\left(X^{\prime} ; R\right)$ are modules in $\mathcal{C}$. Further let $\boldsymbol{F}=(F:(W \partial W)$ $\left.\rightarrow(I \times Y, \partial I \times Y), B: T(W) \oplus\left(p_{Y} \circ F\right)^{*} \eta \rightarrow\left(p_{Y} \circ F\right)^{*}\left(\varepsilon_{Y}(\mathbb{R}) \oplus \xi \oplus \eta\right)\right)$ be an admissible, $G$-framed cobordism between $\boldsymbol{f}$ and $\boldsymbol{f}^{\prime}$. Then $\boldsymbol{F}$ is $G$-framed cobordant rel. $X \cup \operatorname{Sing}(G, W)$ (by $G$-surgery of dimension $\leqq k-1$ and $k$-dimensional handle subtraction in $\operatorname{Free}(G, W))$ to a $k$-connected, admissible, $G$-framed cobordism

$$
\begin{aligned}
& \boldsymbol{F}^{\prime}=\left(F^{\prime}:\right.\left(W^{\prime}, \partial W^{\prime}\right) \rightarrow(I \times Y, \partial I \times Y), \\
&\left.B^{\prime}: T(W) \oplus\left(p_{Y} \circ F^{\prime}\right)^{*} \eta \rightarrow\left(p_{Y} \circ F^{\prime}\right)^{*}\left(\varepsilon_{Y}(\mathbb{R}) \oplus \xi \oplus \eta\right)\right)
\end{aligned}
$$

such that for the degree $1, G$-framed map $f^{\prime \prime}=\left(f^{\prime \prime}: X^{\prime \prime} \rightarrow Y, b^{\prime \prime}: T\left(X^{\prime \prime}\right) \oplus f^{\prime \prime *} \eta \rightarrow\right.$ $\left.f^{\prime \prime *}(\xi \oplus \eta)\right)$ obtained by

$$
\partial \boldsymbol{F}^{\prime}=(-\boldsymbol{f}) \coprod \boldsymbol{f}^{\prime \prime}
$$

the following hold:

(3.1.1) $f^{\prime \prime}: X^{\prime \prime} \rightarrow Y$ is $k$-connected,

(3.1.2) $K_{k}\left(W^{\prime}, \partial W^{\prime} ; \mathbb{Z}\right)=0=K_{k+1}\left(W^{\prime} ; \mathbb{Z}\right)$ and

(3.1.3) $\boldsymbol{M}_{f^{\prime \prime}} \cong \boldsymbol{M}_{f^{\prime}} \oplus \mathbb{M}\left(x_{1}, y_{1}, \beta_{1}\right) \oplus \cdots \oplus \mathbb{M}\left(x_{\ell}, y_{\ell}, \beta_{\ell}\right)$ in $\boldsymbol{S} \boldsymbol{Q}^{\lambda}(\boldsymbol{A}, \Theta(G, X, k))_{\mathcal{C}}$, where $\boldsymbol{A}=\left(R, G, Q, S,(-1)^{k}, w_{X}\right)$ (see $\left.\S 2\right)$, for some nonnegative integer $\ell$ and $\beta_{i}=\left(c_{i}, r_{i}\right), i=1, \ldots, \ell$, consisting of $c_{i}: \Theta(G, X, k) \rightarrow \mathbb{Z}$ and $r_{i}: S(G, X) \rightarrow \mathbb{Z}$ such that $c_{i}(\gamma) \equiv r_{i}(g) \bmod 2 \mathbb{Z}$ for $\gamma \in \Theta(G, X, k)$ and $g \in S(G, X)$ with $g \in \rho(\gamma)$.

In the lemma, $\mathbb{M}\left(x_{i}, y_{i}, \beta_{i}\right)$ are the metabolic planes defined in [3, Definition 5.1] (cf. [3. Lemma 5.5]). The lemma will be proven in Section 4.

Theorem 3.2. Let $R=\mathbb{Z}_{(m)}\left(m=0,1\right.$ or a prime), let $X, X^{\prime} \in \boldsymbol{M} \boldsymbol{n} \boldsymbol{f}_{\mathrm{cl}, \mathrm{sg}}^{n}(G)_{0}$ and $Y \in \boldsymbol{M} \boldsymbol{n} \boldsymbol{f}_{\mathrm{cl}}^{n}(G)_{1}$ with $n=2 k \geqq 6$ even, and let $\boldsymbol{f}=(f: X \rightarrow Y, b$ : $\left.T(X) \oplus f^{*} \eta \rightarrow f^{*}(\xi \oplus \eta)\right)$ and $f^{\prime}=\left(f^{\prime}: X^{\prime} \rightarrow Y, b^{\prime}: T\left(X^{\prime}\right) \oplus f^{\prime *} \eta \rightarrow f^{\prime *}(\xi \oplus \eta)\right)$ be degree $1, k$-connected, $G$-framed maps such that $K_{k}(X ; R)$ and $K_{k}\left(X^{\prime} ; R\right)$ are modules in $\mathcal{C}$. If there exists an admissible, $G$-framed cobordism $\boldsymbol{F}=(F, B)$ between $\boldsymbol{f}$ and $\boldsymbol{f}^{\prime}$ such that $F: W \rightarrow I \times Y$ satisfies the $\mathcal{F}_{m}$-Condition (or the $\mathcal{P}_{0}$-Condition and $\left.\widetilde{K}_{0}(\mathbb{Z}[G])=0\right)$, then $\sigma(\boldsymbol{f})=\sigma\left(\boldsymbol{f}^{\prime}\right)$ in $W_{n}(\boldsymbol{A}, \Theta(G, X, k))_{\mathcal{C}}$.

For the definition of $W_{n}(\boldsymbol{A}, \Theta(G, X, k))_{\mathcal{C}}$, see [3, p.280, (4.4)].

Proof. By Lemma 3.1, there exist a degree 1, G-framed map $\boldsymbol{f}^{\prime \prime}$ and a cobordism $\boldsymbol{F}^{\prime} \in \boldsymbol{C o b}\left(G ; \boldsymbol{f}, \boldsymbol{f}^{\prime \prime}\right)$ satisfying the conditions (3.1.1)-(3.1.3). The equality $\sigma\left(\boldsymbol{f}^{\prime}\right)=$ $\sigma\left(\boldsymbol{f}^{\prime \prime}\right)$ in $W_{n}(\boldsymbol{A}, \Theta(G, X, k))_{\mathcal{C}}$ is clear from (3.1.3). Since $K_{k}\left(W^{\prime} ; R\right)$ is stably free over $R[G]$ (cf. Remark 2.3), the universal coefficient theorem and the PoincaréLefschetz duality theorem guarantee that $K_{k+1}\left(W^{\prime}, \partial W^{\prime} ; R\right)$ is also stably free over $R[G]$. In addition, it follows that

$$
\begin{aligned}
& K_{k}\left(\partial W^{\prime} ; R\right) \cong K_{k}\left(W^{\prime} ; R\right) \oplus K_{k+1}\left(W^{\prime}, \partial W^{\prime} ; R\right), \\
& \operatorname{rank}_{R[G]} K_{k+1}\left(W^{\prime}, \partial W^{\prime} ; R\right)=\operatorname{rank}_{R[G]} K_{k}\left(W^{\prime} ; R\right) .
\end{aligned}
$$


Thus, we can deduce from (2.7.2) and (2.7.3) that $K_{k+1}\left(W^{\prime}, \partial W^{\prime} ; R\right)$ is a stably free Lagrangian of $\overline{\boldsymbol{M}}_{\partial \boldsymbol{F}^{\prime}}$, and hence

$$
\left[\overline{\boldsymbol{M}}_{\partial \boldsymbol{F}^{\prime}}\right]=0 \in W_{n}(\boldsymbol{A}, \Theta(G, X, k))_{((\mathrm{s}-\text { free }))}=W_{n}(\boldsymbol{A}, \Theta(G, X, k))_{((\text {free }))} .
$$

By (2.7.1), we get $\sigma(\boldsymbol{f})=\sigma\left(\boldsymbol{f}^{\prime \prime}\right)$ in $W_{n}(\boldsymbol{A}, \Theta(G, X, k))_{\mathcal{C}}$.

Let $R=\mathbb{Z}_{(m)}$, let $X, X^{\prime} \in \boldsymbol{M} \boldsymbol{n} \boldsymbol{f}_{\mathrm{cl}, \mathrm{sg}}^{n}(G)_{0}$ and $Y \in \boldsymbol{M} \boldsymbol{n} \boldsymbol{f}_{\mathrm{cl}}^{n}(G)_{1}$, where $n=$ $2 k \geqq 6$, and let $\boldsymbol{f}=(f, b)$ be a degree $1, G$-framed map from $X$ to $Y$. Suppose that $f: X \rightarrow Y$ satisfies the $\mathcal{F}_{m}$-Condition (or the $\mathcal{P}_{0}$-Condition and $\widetilde{K}_{0}(\mathbb{Z}[G])=0$ ). Since $\operatorname{dim} \operatorname{Sing}(G, X) \leqq k, \boldsymbol{f}$ is $G$-framed cobordant rel. $\operatorname{Sing}(G, X)$ to $\boldsymbol{f}^{\prime}=\left(f^{\prime}, b^{\prime}\right)$ such that $f^{\prime}: X^{\prime} \rightarrow Y$ is $k$-connected. (Such a $G$-framed cobordism can be obtained by $G$-surgery of dimension $\leqq k-1$ on $\operatorname{Free}(G, X)$.) Then by the $\mathcal{F}_{m}$-Condition (or the $\mathcal{P}_{0}$-Condition and $\left.\widetilde{K}_{0}(\mathbb{Z}[G])=0\right), K_{k}\left(X^{\prime} ; R\right)$ is stably free over $R[G]$. By 3 Lemma 7.1], we obtain the element

$$
\sigma\left(\boldsymbol{f}^{\prime}\right)=\left[\boldsymbol{M}_{\boldsymbol{f}^{\prime}}\right] \in W_{n}(\boldsymbol{A}, \Theta(G, X, k))_{((\text {free }))} .
$$

Now define

$$
\sigma(\boldsymbol{f}):=\sigma\left(\boldsymbol{f}^{\prime}\right) \in W_{n}(\boldsymbol{A}, \Theta(G, X, k))_{((\text {free }))} .
$$

Theorem 3.2 guarantees that $\sigma(\boldsymbol{f})$ is defined independently of the choice of $\boldsymbol{f}^{\prime}$.

Theorem 3.3. Let $R=\mathbb{Z}_{(m)}$, where $m=0,1$ or a prime, and let $X, X^{\prime} \in$ $\boldsymbol{M n} \boldsymbol{f}_{\mathrm{cl}, \mathrm{sg}}^{n}(G)_{0}$ and $Y \in \boldsymbol{M n} \boldsymbol{f}_{\mathrm{cl}}^{n}(G)_{1}$ with $n=2 k \geqq 6$ even, and let $\boldsymbol{f}=(f: X \rightarrow$ $\left.Y, b: T(X) \oplus f^{*} \eta \rightarrow f^{*}(\xi \oplus \eta)\right)$ be a degree 1, G-framed map such that $f: X \rightarrow Y$ satisfies the $\mathcal{F}_{m}$-Condition (or the $\mathcal{P}_{0}$-Condition and $\widetilde{K}_{0}(\mathbb{Z}[G])=0$ ) (cf. Remark 2.3). Then $\sigma(\boldsymbol{f})=0$ in $W_{n}(\boldsymbol{A}, \Theta(G, X, k))_{((\text {free }))}$ if and only if $\boldsymbol{f}$ can be converted by $G$-surgery of isotropy type $\{1\}$ and of dimension $\leqq k$ to a degree $1, k$-connected, G-framed map $\boldsymbol{f}^{\prime}=\left(f^{\prime}: X^{\prime} \rightarrow Y, b^{\prime}: T\left(X^{\prime}\right) \oplus f^{\prime *} \eta \rightarrow f^{\prime *}(\xi \oplus \eta)\right)$ (thus $\boldsymbol{f} \sim \boldsymbol{f}^{\prime}$ rel. $\operatorname{Sing}(G, X))$ such that $f^{\prime}: X^{\prime} \rightarrow Y$ is an $R$-homology equivalence.

Proof. The 'if' part of the claim is obvious from the definition of $\sigma(\boldsymbol{f})$. We have to prove the 'only if' part, namely $\sigma(\boldsymbol{f})=0 \Longrightarrow \boldsymbol{f} \sim \boldsymbol{f}^{\prime}$ rel. $\operatorname{Sing}(G, X)$ as in the claim. Since $\operatorname{dim} \operatorname{Sing}(G, X) \leqq k$, there exists a $k$-connected, degree $1, G$-framed map $\boldsymbol{f}^{\prime \prime}=\left(f^{\prime \prime}: X^{\prime \prime} \rightarrow Y, b^{\prime \prime}: T\left(X^{\prime \prime}\right) \oplus f^{\prime \prime *} \eta \rightarrow f^{\prime \prime *}(\xi \oplus \eta)\right)$ such that $\boldsymbol{f} \sim \boldsymbol{f}^{\prime \prime}$ rel. $\operatorname{Sing}(G, X)$ by $G$-surgery of dimension $\leqq k-1$. Apply Theorem 7.3 of $[3]$ to $f^{\prime \prime}$ and obtain a desired $\boldsymbol{f}^{\prime}$.

\section{Proof of Lemma 3.1}

In this section we prove Lemma 3.1 using equivariant handle subtraction.

First, using Lemma 2.5, we adjust $\boldsymbol{F}$ so that $F: W \rightarrow I \times Y$ is $k$-connected. Fix a small, closed, $n$-dimensional disk $D_{0}$ in general position of Free $\left(G, X^{\prime}\right)$. Thus we may assume that $D_{0} \cap g D_{0}=\emptyset$ if $g \in G \backslash\{1\}$. For each $\gamma \in \Theta(G, X, k)=$ $\Theta\left(G, X^{\prime}, k\right)$, let us take a point $x_{\gamma} \in X_{\gamma}^{\prime}$ such that $G_{x_{\gamma}}=\rho_{X^{\prime}}^{G}\left(X_{\gamma}^{\prime}\right)(=\rho(\gamma))$ and small closed, $n$-dimensional, $G_{x_{\gamma}}$-invariant disks $D_{\gamma}$ with center $x_{\gamma}$ in $X^{\prime} \backslash G D_{0}$ so that $g D_{\gamma} \cap g^{\prime} D_{\gamma^{\prime}}=\emptyset$ unless $\gamma=\gamma^{\prime}$ and $g G_{x_{\gamma}}=g^{\prime} G_{x_{\gamma^{\prime}}}$ (i.e. $g x_{\gamma}=g^{\prime} x_{\gamma^{\prime}}$ ), where $\gamma$ and $\gamma^{\prime}$ range over $\Theta(G, X, k)$ and $g$ and $g^{\prime}$ range over $G$. Here $G D_{\gamma}$ becomes a $G$-equivariant tubular neighborhood of $G x_{\gamma}$. Take connecting tubes $T_{\gamma}$ from $\partial D_{0}$ to $\partial D_{\gamma}$ in general position of

$$
\operatorname{Free}\left(G, X^{\prime}\right) \backslash G\left(\operatorname{Int}\left(D_{0}\right)\right) \backslash \bigcup_{\gamma \in \Theta(G, X, k)} G\left(\operatorname{Int}\left(D_{\gamma}\right)\right) .
$$




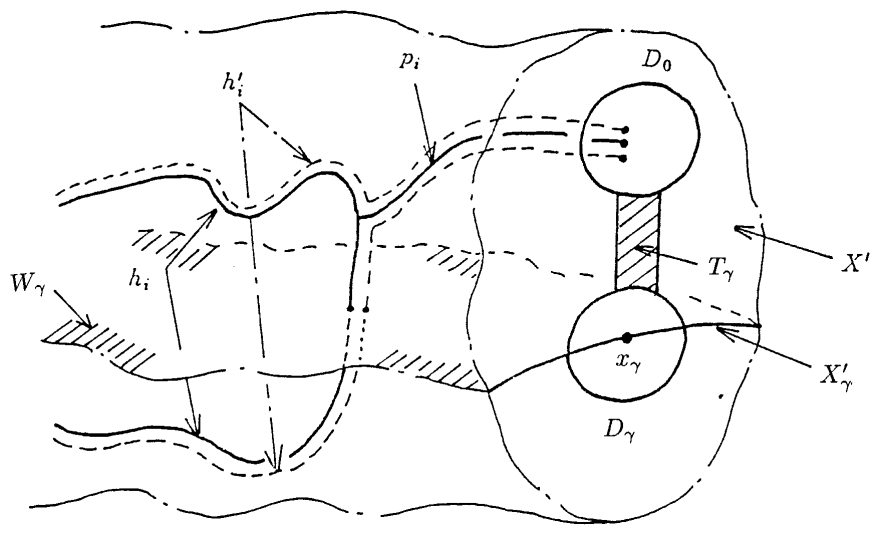

Figure 4.1.

Then,

$$
D:=D_{0} \cup \bigcup_{\gamma \in \Theta(G, X, k)}\left(D_{\gamma} \cup T_{\gamma}\right)
$$

is homeomorphic to the $n$-dimensional disk $D^{n}$. Since $K_{k}(W ; \mathbb{Z}) \rightarrow K_{k}(W, \partial W ; \mathbb{Z})$ is surjective, we can take finitely many embeddings $h_{i}: S^{k} \rightarrow \operatorname{Int}(W), i=1, \cdots, \ell$, say, which together generate $K_{k}(W, \partial W ; \mathbb{Z})$. Without loss of generality, we can assume that $g h_{i}$ and $g^{\prime} h_{j}$ are disjoint unless $i=j$. For each $i$, take a path

$$
p_{i}:[0,1] \hookrightarrow \operatorname{Free}(G, W)
$$

in general position, such that for $t \in[0,1], p_{i}(t) \in X^{\prime} \Leftrightarrow t=0$ and $p_{i}(t) \in \operatorname{Im}\left(h_{i}\right) \Leftrightarrow$ $t=1$, and $p_{i}(0) \in \operatorname{Int} D_{0}$. Choose a thin band $u_{i}:[0,1] \times D^{k} \hookrightarrow$ Free $(G, W)$ along the path $p_{i}$ (i.e., $u_{i}(t, 0)=p_{i}(t)$ for all $\left.t \in[0,1]\right)$ such that for $t \in[0,1]$ and $z \in D^{k}$, $u_{i}(t, z) \in X^{\prime} \Leftrightarrow t=0$ and $u_{i}(t, z) \in \operatorname{Im}\left(h_{i}\right) \Leftrightarrow t=1$, and $u_{i}\left(0, D^{k}\right) \subset \operatorname{Int}\left(D_{0}\right)$. Then,

$$
u_{i}\left([0,1] \times S^{k-1}\right) \cup \operatorname{Im}\left(h_{i}\right) \backslash u_{i}\left(1, \operatorname{Int}\left(D^{k}\right)\right)
$$

gives an embedding $h_{i}^{\prime}:\left(D^{k}, S^{k-1}\right) \rightarrow\left(W, \operatorname{Free}\left(G, X^{\prime}\right)\right)$. (See Figure 4.1.)

Here the restriction $\partial h^{\prime}{ }_{i}: S^{k-1} \rightarrow \operatorname{Free}\left(G, X^{\prime}\right)$ of $h^{\prime}{ }_{i}$ is a trivial embedding close to $p_{i}(0)$. We may suppose that $g h^{\prime}{ }_{i}$ and $g^{\prime} h^{\prime}{ }_{j}$ are disjoint unless $i=j$. For $\gamma \in \Theta(G, X, k)$, let $c_{i}(\gamma)$ denote the ordinary intersection number of $W_{\gamma}$ and $\operatorname{Im}\left(h^{\prime}{ }_{i}\right)$. Thus we obtain a map $c_{i}: \Theta(G, X, k) \rightarrow \mathbb{Z}$. Without loss of generality, we can assume that for $\gamma \in \Theta(G, X, k), h_{i}$ meets with $W_{\gamma}$ at distinct $m$-points $A(i, \gamma, 1), \cdots, A(i, \gamma, m)$ of isotropy subgroup $\rho(\gamma)$, where $m=\left|c_{i}(\gamma)\right|$. Take distinct points $B(i, \gamma, 1), \cdots, B(i, \gamma, m)$ in $h_{i}^{\prime}\left(S^{k-1}\right)$ and $C(i, \gamma, 1), \cdots, C(i, \gamma, m)$ in $\operatorname{Int}\left(D_{\gamma}\right) \cap X_{\gamma}^{\prime=\rho(\gamma)}$. Furthermore take disjoint embedded triangles $\triangle(i, \gamma, j)=$ $\triangle A(i, \gamma, j) B(i, \gamma, j) C(i, \gamma, j)$ (which is homeomorphic to $D^{2}$ ) in $W$ so that

$$
\begin{aligned}
& \overline{A(i, \gamma, j) B(i, \gamma, j)} \subset \operatorname{Im}\left(h_{i}^{\prime}\right), \\
& \overline{A(i, \gamma, j) C(i, \gamma, j)} \subset W_{\gamma}^{=\rho(\gamma)}, \\
& \overline{B(i, \gamma, j) C(i, \gamma, j)} \subset D .
\end{aligned}
$$




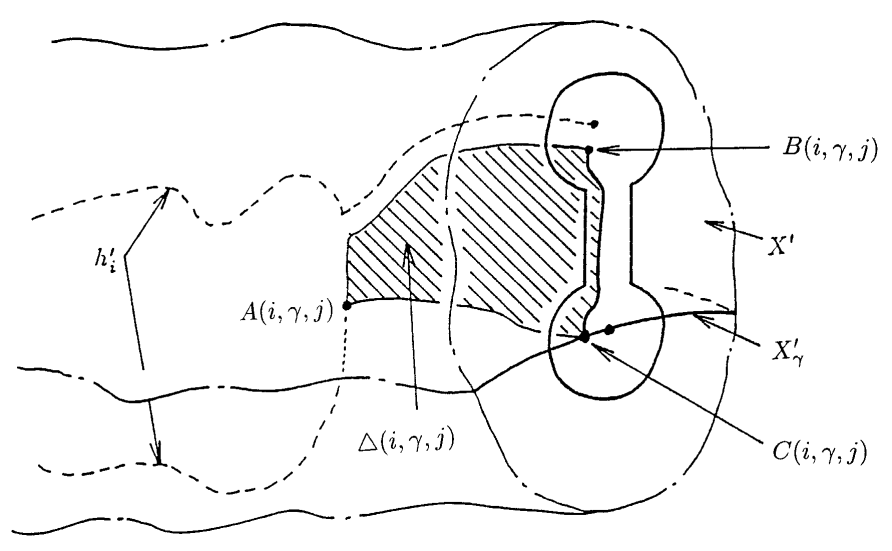

Figure 4.2 .

We may assume that $g(\operatorname{Int}(\triangle(i, \gamma, j))) \cap g^{\prime}\left(\operatorname{Int}\left(\triangle\left(i^{\prime}, \gamma^{\prime}, j^{\prime}\right)\right)\right)=\emptyset$ unless $\gamma=\gamma^{\prime}$, $j=j^{\prime}, i=i^{\prime}$ and $g=g^{\prime}$. Moreover we may assume that $\triangle(i, \gamma, j)$ is perpendicular to $W_{\gamma}, X^{\prime}$ and $\operatorname{Im}\left(h_{i}^{\prime}\right)$. For each $i$, delete the intersection points $A(i, \gamma, j)$ of $h_{i}^{\prime}$ with $W_{\gamma}$ along the triangle $\triangle(i, \gamma, j)$ by [8. Lemma 1.2], where $\gamma \in \Theta(G, X, k)$ and $j=1, \cdots, m$, and obtain an embedding

$$
h_{i}^{\prime \prime}:\left(D^{k}, S^{k-1}\right) \rightarrow\left(\operatorname{Free}(G, W), \operatorname{Free}\left(G, X^{\prime}\right)\right) .
$$

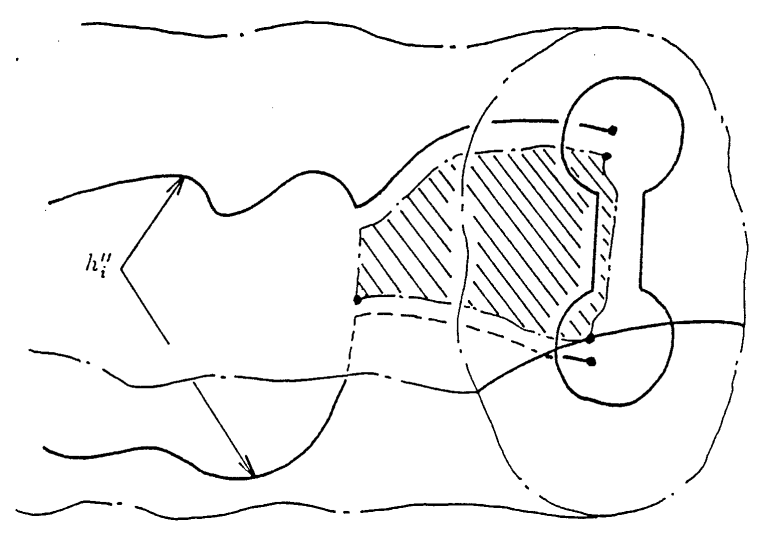

Figure 4.3 .

Let $c: \Theta(G, X, k) \rightarrow \mathbb{Z}$ be a map. Applying the construction of [3, p.298, $\ell .-10$ - p.299, $\ell .18]$ to the data $X$ replaced by $X^{\prime}, S_{c+}=\{s \in S \mid c(s)>0\}$, $S_{c-}=\{s \in S \mid c(s)<0\}, S_{c}^{\prime}=S_{c+} \cup S_{c-}$ and $\Gamma_{c}=\left\{(s, c)\left|s \in S^{\prime}, 1 \leqq i \leqq\right| c(s) \mid\right\}$, we obtain an embedding

$$
\begin{aligned}
& h_{D_{c}}:\left(D^{k} \cong\right) D_{c} \rightarrow X^{\prime}, \\
& h_{c}=\left.h_{D_{c}}\right|_{\partial D_{c}}:\left(S^{k-1} \cong\right) \partial D_{c} \rightarrow \operatorname{Free}\left(G, X^{\prime}\right) .
\end{aligned}
$$

With this notation, the restriction $\partial h_{i}^{\prime \prime} \mid: S^{k-1} \rightarrow \operatorname{Free}\left(G, X^{\prime}\right)$ of $h_{i}^{\prime \prime}$ is the connected sum of $\partial h_{i}^{\prime}$ and $h_{c_{i}}$. 
Thickening $h_{i}^{\prime \prime}$, we obtain embeddings

$$
H_{i}^{\prime \prime}:\left(D^{k} \times D^{k+1}, S^{k-1} \times D^{k+1}\right) \rightarrow\left(\operatorname{Free}(G, W), \text { Free }\left(G, X^{\prime}\right)\right), \quad i=1, \cdots, \ell .
$$

Now we may suppose that $g H_{i}^{\prime \prime}$ and $g^{\prime} H_{j}^{\prime \prime}$ are disjoint unless $g=g^{\prime}$ and $i=j$. Further we may suppose that $F\left(\operatorname{Im}\left(H_{i}^{\prime \prime}\right)\right)$ is a point in $\{1\} \times Y$. Set

$$
\begin{aligned}
& V=\bigcup_{i=1}^{\ell} G\left(\operatorname{Im}\left(H_{i}^{\prime \prime}\right)\right), \\
& \partial_{+} V=\bigcup_{i=1}^{\ell} G\left(H_{i}^{\prime \prime}\left(S^{k-1} \times D^{k+1}\right)\right), \\
& W^{\prime}=\operatorname{Closure}(W \backslash V), \\
& X^{\prime \prime}=\left(X^{\prime} \cup V\right) \backslash \bigcup_{i=1}^{\ell} G\left(H_{i}^{\prime \prime}\left(D^{k} \times \operatorname{Int}\left(D^{k+1}\right)\right)\right), \\
& F^{\prime}=\left.F\right|_{W^{\prime}}: W^{\prime} \rightarrow I \times Y, \\
& B^{\prime}=\left.B\right|_{W^{\prime}}: T\left(W^{\prime}\right) \oplus\left(p_{Y} \circ F^{\prime}\right)^{*} \eta \rightarrow\left(p_{Y} \circ F^{\prime}\right)^{*}\left(\varepsilon_{Y}(\mathbb{R}) \oplus \xi \oplus \eta\right), \\
& f^{\prime \prime}=\left.F\right|_{X^{\prime \prime}}: X^{\prime \prime} \rightarrow\{1\} \times Y=Y .
\end{aligned}
$$

By [3, Proposition 10.1], we may assume that $B^{\prime}$ has on $X^{\prime \prime}$ the form

$$
i d_{X^{\prime \prime}} \oplus b^{\prime \prime}=\left.B^{\prime}\right|_{X^{\prime \prime}}: \varepsilon_{X^{\prime \prime}}(\mathbb{R}) \oplus T\left(X^{\prime \prime}\right) \oplus{f^{\prime \prime *}}^{*} \rightarrow \varepsilon_{X^{\prime \prime}}(\mathbb{R}) \oplus f^{\prime \prime *}(\xi \oplus \eta) .
$$

It remains to check that the above $\boldsymbol{F}^{\prime}=\left(F^{\prime}, B^{\prime}\right)$ and $\boldsymbol{f}^{\prime \prime}=\left(f^{\prime \prime}, b^{\prime \prime}\right)$ are $G$-framed maps as desired in Lemma 3.1. By excision, $K_{k}\left(W^{\prime}, \partial W^{\prime} ; \mathbb{Z}\right)=K_{k}(W, \partial W \cup V ; \mathbb{Z})$. There is an exact sequence:

$$
K_{k}(\partial W \cup V, \partial W ; \mathbb{Z}) \rightarrow K_{k}(W, \partial W ; \mathbb{Z}) \rightarrow K_{k}(W, \partial W \cup V ; \mathbb{Z}) \rightarrow 0 .
$$

Since the first arrow is surjective, $K_{k}(W, \partial W \cup V ; \mathbb{Z})=0$. Thus we get

$$
K_{k}\left(W^{\prime}, \partial W^{\prime} ; \mathbb{Z}\right)=0 .
$$

By the universal coefficient theorem, $K^{k}\left(W^{\prime}, \partial W^{\prime} ; \mathbb{Z}\right)=0$. The Poincaré-Lefschetz duality implies $K_{k+1}\left(W^{\prime} ; \mathbb{Z}\right)=0$. Note that $\boldsymbol{f}^{\prime \prime}$ can be obtained from $\boldsymbol{f}^{\prime}$ by $G$ surgery along the embeddings $\partial h_{1}^{\prime \prime}, \cdots, \partial h_{\ell}^{\prime \prime}: S^{k-1} \rightarrow X^{\prime}$. These embeddings are isotopic in general position of $X^{\prime}$ to $h_{c_{1}}, \cdots, h_{c_{\ell}}$, respectively. By the same

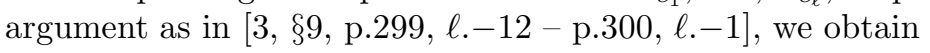

$$
\boldsymbol{M}_{\boldsymbol{f}^{\prime \prime}} \cong \boldsymbol{M}_{\boldsymbol{f}^{\prime}} \oplus \mathbb{M}\left(x_{1}, y_{1}, \beta_{1}\right) \oplus \cdots \oplus \mathbb{M}\left(x_{\ell}, y_{\ell}, \beta_{\ell}\right),
$$

and complete the proof.

\section{REFERENCES}

[1] A. Bak and M. Morimoto, Equivariant surgery on compact manifolds with half dimensional singular sets, Preprint (1992).

[2] A. Bak and M. Morimoto, $K$-theoretic groups with positioning map and equivariant surgery, Proc. Japan Acad. 70 A (1994), 6-11. MR 95e:19006

[3] A. Bak and M. Morimoto, Equivariant surgery with middle dimensional singular sets. I, Forum Math. 8 (1996), 267-302. MR 97b:57031

[4] N. P. Buchdahl, S. Kwasik and R. Schultz, One fixed point actions on low-dimensional spheres, Invent. Math. 102 (1990), 633-662. MR 92b:57047

[5] K. H. Dovermann, $\mathbb{Z}_{2}$ surgery theory, Michigan Math. J. 28 (1981), 267-287. MR 83b:57019

[6] K. H. Dovermann and R. Schultz, Surgery of involutions with middle-dimensional fixed point set, Pacific J. Math. 130 (1987), 275-297. MR 89e:57032 
[7] E. Laitinen and M. Morimoto, Finite groups with smooth one fixed point actions on spheres, Forum Math. 10 (1998), 479-520. MR 99k:57078

[8] M. Morimoto, Bak groups and equivariant surgery II, K-Theory 3 (1990), 505-521. MR 91g:57034

[9] M. Morimoto, Equivariant surgery theory: Construction of equivariant normal maps, Publ. Res. Inst. Math. Sci. Kyoto Univ. 31 (1995), 145-167. MR 96a:57077

[10] C.T.C. Wall, Surgery on Compact Manifolds, Academic Press, London, 1970. MR 55:4217

Department of Environmental and Mathematical Sciences, Faculty of Environmental Science and Technology, Okayama University, Tsushimanaka 3-1-1, Okayama, 7008530 JAPAN

E-mail address: morimoto@math.ems.okayama-u.ac.jp 